Supplement of Nat. Hazards Earth Syst. Sci., 21, 1599-1614, 2021

https://doi.org/10.5194/nhess-21-1599-2021-supplement

(C) Author(s) 2021. CC BY 4.0 License.

(c) (i)

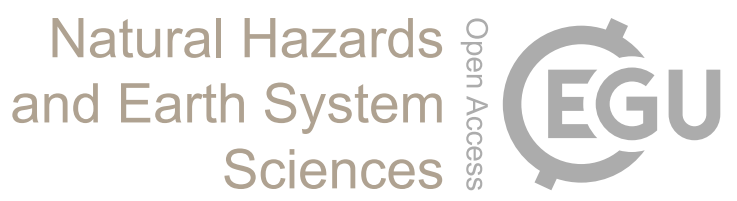

Supplement of

\title{
Residential flood loss estimated from Bayesian multilevel models
}

\section{Guilherme S. Mohor et al.}

Correspondence to: Guilherme S. Mohor (samprognamoh@uni-potsdam.de)

The copyright of individual parts of the supplement might differ from the article licence. 


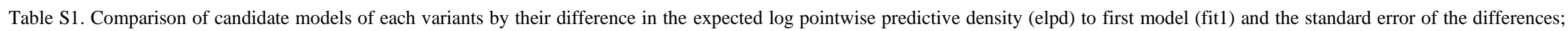
model candidates with cumulative predictors from 1 to 12

\begin{tabular}{|c|c|c|c|c|c|c|c|c|c|c|c|c|c|c|c|c|}
\hline \multicolumn{4}{|c|}{ Single-level } & \multicolumn{4}{|c|}{ Flood Type } & \multicolumn{4}{|l|}{ Region } & \multicolumn{4}{|l|}{ Event } & \multirow[b]{2}{*}{ Predictors } \\
\hline Model & $\begin{array}{l}\text { elpd_ } \\
\text { loo }\end{array}$ & $\begin{array}{l}\text { elpd__ } \\
\text { diff }\end{array}$ & $\begin{array}{l}\mathrm{se}_{-} \\
\text {diff }\end{array}$ & Model & $\begin{array}{l}\text { elpd } \\
\text { loo }\end{array}$ & $\begin{array}{l}\text { elpd_ } \\
\text { diff }\end{array}$ & $\begin{array}{l}\mathrm{se}_{-} \\
\text {diff }\end{array}$ & Model & $\begin{array}{l}\text { elpd_ } \\
\text { loo }\end{array}$ & $\begin{array}{l}\text { elpd_ } \\
\text { diff }\end{array}$ & $\begin{array}{l}\mathrm{se}_{-} \\
\text {diff }\end{array}$ & Model & $\begin{array}{l}\text { elpd_ } \\
\text { loo }\end{array}$ & $\begin{array}{l}\text { elpd_ } \\
\text { diff }\end{array}$ & $\begin{array}{l}\mathrm{se}_{-} \\
\text {diff }\end{array}$ & \\
\hline fit1 & 1986.4 & 0 & 0 & fit1 & 2018.68 & 0 & 0 & fit1 & 2002.60 & 0 & 0 & fit1 & 2036.28 & 0 & 0 & WD \\
\hline fit2 & 2033.8 & 47.34 & 9.56 & fit2 & 2057.33 & 38.65 & 8.72 & fit2 & 2041.76 & 39.16 & 8.60 & fit2 & 2074.17 & 37.89 & 8.55 & $\mathrm{WD}+\mathrm{BA}$ \\
\hline fit3 & 2079.6 & 93.15 & 13.88 & fit3 & 2093.22 & 74.54 & 12.45 & fit3 & 2085.28 & 82.67 & 13.14 & fit3 & 2109.95 & 73.67 & 13.26 & $\mathrm{WD}+\mathrm{BA}+\mathrm{Con}$ \\
\hline fit4 & 2092.3 & 105.81 & 15.3 & fit4 & 2098.06 & 79.37 & 12.84 & fit4 & 2094.31 & 91.71 & 14.49 & fit 4 & 2122.40 & 86.13 & 14.51 & $\mathrm{WD}+\mathrm{BA}+\mathrm{Con}+\mathrm{Dur}$ \\
\hline fit5 & 2109.8 & 123.33 & 16.11 & fit5 & 2113.37 & 94.69 & 13.62 & fit5 & 2107.96 & 105.36 & 15.14 & fit5 & 2126.39 & 90.11 & 14.95 & $\mathrm{WD}+\mathrm{BA}+\mathrm{Con}+\mathrm{Dur}+\mathrm{Pre}$ \\
\hline fit6 & 2118.1 & 131.61 & 16.41 & fit6 & 2124.02 & 105.34 & 14.10 & fit6 & 2111.94 & 109.33 & 15.50 & fit6 & 2130.38 & 94.11 & 15.46 & WD+BA+Con+Dur+Pre+Ins \\
\hline fit7 & 2122.9 & 136.47 & 16.68 & fit $7 *$ & 2127.00 & 108.32 & 14.50 & fit7 & 2115.93 & 113.32 & 15.68 & fit7* & 2132.51 & 96.23 & 15.72 & WD+BA+Con+Dur+Pre+Ins+Eff \\
\hline fit8* & 2122.0 & 135.54 & 16.65 & fit8* & 2125.44 & 106.76 & 14.53 & fit8* & 2115.38 & 112.78 & 15.66 & fit8* & 2130.47 & 94.19 & 15.69 & WD+BA+Con+Dur+Pre+Ins+Eff+Eme \\
\hline fit $9 *$ & 2124.0 & 137.5 & 16.93 & fit $9 *$ & 2126.19 & 107.51 & 14.82 & fit $9 *$ & 2117.35 & 114.75 & 16.19 & fit $9 *$ & 2132.64 & 96.36 & 15.43 & WD+BA+Con+Dur+Pre+Ins+Eff+Eme+Cel \\
\hline fit $10^{*}$ & 2125.1 & 138.69 & 16.88 & fit $10^{*}$ & 2125.88 & 107.20 & 14.78 & fit $10^{*}$ & 2120.50 & 117.90 & 16.25 & fit10* & 2133.34 & 97.06 & 15.64 & WD+BA+Con+Dur+Pre+Ins+Eff+Eme+Cel+Vel \\
\hline fit11 & 2130.5 & 144.02 & 16.91 & fit11 & 2131.83 & 113.15 & 15.11 & fit $11^{*}$ & 2122.01 & 119.40 & 16.23 & fit11* & 2133.62 & 97.34 & 15.61 & WD+BA+Con+Dur+Pre+Ins+Eff+Eme+Cel+Vel+Exp \\
\hline fit12 & 2134.6 & 148.14 & 16.99 & fit $12 *$ & 2134.26 & 115.58 & 15.26 & fit $12 *$ & 2125.23 & 122.62 & 16.26 & fit12* & 2134.36 & 98.08 & 15.72 & $\mathrm{WD}+\mathrm{BA}+\mathrm{Con}+\mathrm{Dur}+\mathrm{Pre}+\mathrm{Ins}+\mathrm{Eff}+\mathrm{Eme}+\mathrm{Cel}+\mathrm{Vel}+\mathrm{Exp}+\mathrm{BQ}$ \\
\hline
\end{tabular}

* model complexity after which little gain is observed (elpd_diff <4) 


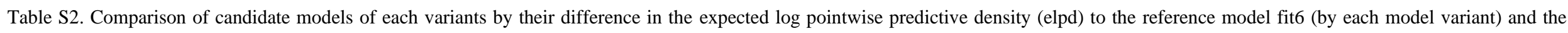
standard error of the differences; model candidates with predictors 1 to 6 plus one of the remaining predictors

\begin{tabular}{|c|c|c|c|c|c|c|c|c|c|c|c|c|c|c|c|c|}
\hline \multicolumn{4}{|c|}{ Single-level } & \multicolumn{4}{|c|}{ Flood Type } & \multicolumn{4}{|l|}{ Region } & \multicolumn{4}{|l|}{ Event } & \multirow{2}{*}{$\begin{array}{c}\text { Predictors } \\
\text { (for Event model variant) }\end{array}$} \\
\hline Model & $\begin{array}{l}\text { elpd } \\
\text { loo }\end{array}$ & \begin{tabular}{|l|} 
elpd__ \\
diff
\end{tabular} & $\begin{array}{l}\mathrm{se}_{-} \\
\text {diff }\end{array}$ & Model & $\begin{array}{l}\text { elpd_ } \\
\text { loo }\end{array}$ & $\begin{array}{l}\text { elpd_- } \\
\text { diff }\end{array}$ & \begin{tabular}{|l} 
se \\
diff
\end{tabular} & Model & $\begin{array}{l}\text { elpd_ } \\
\text { loo }\end{array}$ & $\begin{array}{l}\text { elpd_ } \\
\text { diff }\end{array}$ & $\begin{array}{l}\mathrm{se}_{-} \\
\text {diff }\end{array}$ & Model & $\begin{array}{l}\text { elpd_ } \\
\text { loo }\end{array}$ & $\begin{array}{l}\text { elpd_ } \\
\text { diff }\end{array}$ & $\begin{array}{l}\mathrm{se}_{-} \\
\text {diff }\end{array}$ & \\
\hline fit $6+8$ & 2117.3 & -0.8 & 0.2 & fit6+8 & 2122.3 & -1.7 & 0.5 & fit6+8 & 2111.7 & -0.3 & 1.1 & fit6+8 & 2129.0 & -1.4 & 0.4 & $\mathrm{WD}+\mathrm{BA}+\mathrm{Con}+\mathrm{Dur}+\mathrm{Pre}+\mathrm{Ins}+\mathrm{Eme}$ \\
\hline fit6 & 2118.1 & 0 & 0 & fit6+10 & 2123.2 & -0.9 & 1.4 & fit6 & 2111.9 & 0 & 0 & fit $6+11$ & 2130.0 & -0.3 & 1.7 & $\mathrm{WD}+\mathrm{BA}+\mathrm{Con}+\mathrm{Dur}+\mathrm{Pre}+\mathrm{Ins}+\mathrm{Exp}$ \\
\hline fit6+10 & 2118.9 & 0.8 & 2.1 & fit6 & 2124.0 & 0 & 0 & fit $6+12$ & 2113.2 & 1.2 & 2.4 & fit6 & 2130.4 & 0 & 0 & $\mathrm{WD}+\mathrm{BA}+\mathrm{Con}+\mathrm{Dur}+\mathrm{Pre}+\mathrm{Ins}$ \\
\hline fit6+9 & 2119.9 & 1.9 & 2.6 & fit6+12 & 2124.2 & 0.2 & 2.0 & fit6+9 & 2114.7 & 2.7 & 3.3 & fit6+10 & 2131.1 & 0.7 & 2.1 & $\mathrm{WD}+\mathrm{BA}+\mathrm{Con}+\mathrm{Dur}+\mathrm{Pre}+\mathrm{Ins}+\mathrm{Vel}$ \\
\hline fit6+12 & 2121.3 & 3.3 & 2.9 & fit6+9 & 2124.4 & 0.3 & 2.0 & fit6+11 & 2115.0 & 3.1 & 2.8 & fit6+12 & 2131.2 & 0.8 & 2.1 & $\mathrm{WD}+\mathrm{BA}+\mathrm{Con}+$ Dur+Pre+Ins+BQ \\
\hline fit $6+7 *$ & 2122.9 & 4.9 & 3.6 & fit6+7 & 2127.0 & 3.0 & 3.5 & fit6+10 & 2115.6 & 3.6 & 3.2 & fit6+9 & 2132.1 & 1.7 & 3.0 & $\mathrm{WD}+\mathrm{BA}+\mathrm{Con}+\mathrm{Dur}+\mathrm{Pre}+\mathrm{Ins}+\mathrm{Cel}$ \\
\hline fit6+11* & 2123.0 & 5.0 & 3.3 & fit6+11* & 2130.8 & 6.7 & 3.9 & fit $6+7 *$ & 2115.9 & 4.0 & 3.4 & fit6+7 & 2132.5 & 2.1 & 3.0 & WD+BA+Con+Dur+Pre+Ins+Eff \\
\hline
\end{tabular}

* model with relevant improvement compared to others (elpd_diff $>4$ and elpd_diff $>$ se_diff)

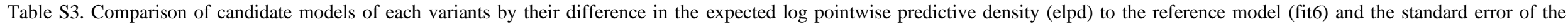
differences; model candidates with predictors 1 to 5 plus a combination of predictors 6,7 , and 11

\begin{tabular}{|c|c|c|c|c|c|c|c|c|c|c|c|c|c|c|c|c|}
\hline \multicolumn{4}{|c|}{ Single-Level } & \multicolumn{4}{|l|}{ Flood Type } & \multicolumn{4}{|l|}{ Region } & \multicolumn{4}{|l|}{ Event } & \multirow{2}{*}{$\begin{array}{c}\text { Predictor } \\
\text { (for Event variant) }\end{array}$} \\
\hline Model & $\begin{array}{l}\text { elpd__ } \\
\text { diff }\end{array}$ & $\begin{array}{l}\mathrm{se}_{-} \\
\text {diff }\end{array}$ & $\begin{array}{l}\text { elpd_ } \\
\text { loo }\end{array}$ & Model & $\begin{array}{l}\text { elpd__ } \\
\text { diff }\end{array}$ & $\begin{array}{l}\mathrm{se}_{-} \\
\text {diff }\end{array}$ & $\begin{array}{l}\text { elpd_ } \\
\text { loo }\end{array}$ & Model & $\begin{array}{l}\text { elpd__ } \\
\text { diff }\end{array}$ & $\begin{array}{l}\mathrm{se}_{-} \\
\text {diff }\end{array}$ & $\begin{array}{l}\text { elpd } \\
\text { loo }\end{array}$ & Model & \begin{tabular}{|l} 
elpd_ \\
diff
\end{tabular} & $\begin{array}{l}\mathrm{se}_{-} \\
\text {diff }\end{array}$ & $\begin{array}{l}\text { elpd_ } \\
\text { loo }\end{array}$ & \\
\hline fit5+7* & -3.6 & 5.7 & 2114.5 & fit $5+7$ & -6.2 & 6.1 & 2117.8 & fit $5+11^{*}$ & -0.9 & 4.2 & 2111.0 & fit $5+11$ & -4.3 & 4.0 & 2126.1 & WD+BA+Con+Dur+Pre+Exp \\
\hline fit $5+11 *$ & -3.1 & 5.5 & 2115.0 & fit $5+11^{*}$ & -3.5 & 6.4 & 2120.5 & fit5+7* & -0.2 & 4.7 & 2111.7 & fit5+7* & -2.3 & 4.6 & 2128.1 & $\mathrm{WD}+\mathrm{BA}+\mathrm{Con}+$ Dur+Pre+Eff \\
\hline fit6* & 0 & 0 & 2118.1 & fit6* & 0 & 0 & 2124.0 & fit6* & 0 & 0 & 2111.9 & fit $5+7+11 *$ & -1.4 & 5.0 & 2129.0 & WD+BA+Con+Dur+Pre+Eff+Exp \\
\hline fit $5+7+11 *$ & 1.6 & 6.7 & 2119.7 & fit $5+7+11 *$ & 0.1 & 7.4 & 2124.1 & fit $5+7+11^{*}$ & 2.3 & 5.5 & 2114.2 & fit $6+11^{*}$ & -0.3 & 1.7 & 2130.0 & WD+BA+Con+Dur+Pre+Ins+Exp \\
\hline fit $6+7$ & 4.9 & 3.5 & 2122.9 & fit6+7* & 3.0 & 3.5 & 2127.0 & fit $6+11^{*}$ & 3.1 & 2.8 & 2115.0 & fit6* & 0 & 0 & 2130.4 & WD+BA+Con+Dur+Pre+Ins \\
\hline fit6+11 & 5.0 & 3.3 & 2123.0 & fit6+11 & 6.7 & 3.9 & 2130.8 & fit $6+7$ & 4.0 & 3.4 & 2115.9 & fit $6+7+11 *$ & 1.5 & 3.5 & 2131.9 & $\mathrm{WD}+\mathrm{BA}+\mathrm{Con}+\mathrm{Dur}+\mathrm{Pre}+\mathrm{Ins}+\mathrm{Eff}+\mathrm{Exp}$ \\
\hline fit $6+7+11$ & 9.6 & 4.8 & 2127.6 & fit6+7+11 & 9.6 & 5.4 & 2133.6 & fit $6+7+11$ & 6.4 & 4.3 & 2118.3 & fit6+7* & 2.1 & 3.0 & 2132.5 & $\mathrm{WD}+\mathrm{BA}+\mathrm{Con}+\mathrm{Dur}+\mathrm{Pre}+\mathrm{Ins}+\mathrm{Eff}$ \\
\hline
\end{tabular}

*models with predictive accuracy that is indistinguishable from that of the reference model fit6 


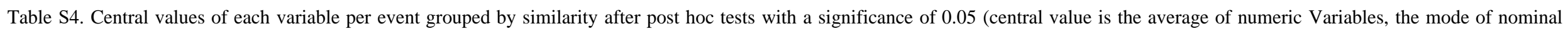
variables)

\begin{tabular}{|c|c|c|c|c|c|c|c|c|c|c|c|c|}
\hline EVENT & 2002 & & 2005 & & 2006 & & 2010 & & 2011 & & 2013 & \\
\hline $\mathbf{n}$ & 1697 & & 305 & & 156 & & 440 & & 218 & & 1652 & \\
\hline Water depth & 64.212 & $\mathrm{a}$ & -19.351 & $\mathrm{~b}$ & 18.816 & $\mathrm{~b}, \mathrm{c}$ & 24.669 & $\mathrm{c}$ & -23.271 & $\mathrm{~b}, \mathrm{c}$ & 53.526 & d \\
\hline Duration & 142.89 & $\mathrm{a}$ & 52.371 & $\mathrm{~b}$ & 146.18 & $\mathrm{c}$ & 57.962 & $\mathrm{~b}$ & 101.21 & $\mathrm{a}$ & 206.04 & $\mathrm{~d}$ \\
\hline Velocity & 32.326 & $\mathrm{a}$ & 29.304 & $a, b$ & 26.533 & $\mathrm{~b}, \mathrm{c}$ & 33.535 & $\mathrm{a}$ & 23.876 & $\mathrm{~b}, \mathrm{c}$ & 24.833 & $\mathrm{c}$ \\
\hline Contamination & 0.67265 & $\mathrm{a}$ & 0.27 & $\mathrm{~b}$ & 0.35099 & $\mathrm{~b}, \mathrm{c}$ & 0.54801 & $\mathrm{~d}$ & 0.25463 & $\mathrm{~b}, \mathrm{c}$ & 0.45702 & $\mathrm{c}$ \\
\hline Loss ratio & 0.12262 & $\mathrm{a}$ & 0.040601 & $\mathrm{~b}$ & 0.069922 & $\mathrm{~b}, \mathrm{c}$ & 0.077278 & $\mathrm{c}$ & 0.019505 & $\mathrm{~d}$ & 0.11732 & $\mathrm{a}$ \\
\hline
\end{tabular}

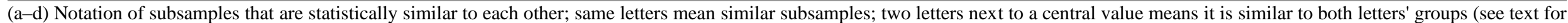
reading example). 